\title{
Palladium-Catalyzed Intramolecular Oxidative Alkylation of Unactivated Olefins
}

Tao Pei, Xiang Wang, and Ross A. Widenhoefer*

P. M. Gross Chemical Laboratory

Duke University

Durham, NC 27708-0346

\section{Supporting Information}

Experimental procedures, analytical and spectroscopic data for new compounds and cyclohexanones (14 pages). 


\section{Experimental}

General Methods. Reactions were performed under an atmosphere of nitrogen employing standard Schlenk techniques unless otherwise stated. NMR were obtained on a Varian spectrometer operating at $400 \mathrm{MHz}$ for ${ }^{1} \mathrm{H} \mathrm{NMR}$ and $100 \mathrm{MHz}$ for ${ }^{13} \mathrm{C} \mathrm{NMR}$ in $\mathrm{CDCl}_{3}$ unless otherwise noted. IR spectra were obtained on a Bomen MB-100 FT IR spectrometer. Gas chromatography was performed on a HP 5890 gas chromatography equipped with a 25 m polydimethylsiloxane capillary column. Flash column chromatography was performed employing 200-400 mesh silica gel (EM); all compounds were isolated as colorless oils unless otherwise noted. Elemental analyses were performed by Complete Analysis Laboratories (Parsippany, NJ). For compounds that exist as mixtures of enol and keto tautomers, the enol:keto ratio for each compound was determined by ${ }^{1} \mathrm{H}$ NMR spectroscopy in $\mathrm{CDCl}_{3}$; only the resonances corresponding to the predominant tautomer are given. Thin layer chromatography (TLC) was performed on $\mathrm{SiO}_{2}$ plates eluting with a 5:1 mixture of hexanes-EtOAc unless noted otherwise. 1,4Dioxane (Aldrich anhydrous) and $\mathrm{PdCl}_{2}\left(\mathrm{CH}_{3} \mathrm{CN}\right)_{2}$ (Strem) were used as received. 1,2-Dichloroethane (DCE) was distilled from $\mathrm{CaH}_{2}$ under nitrogen. 8-Nonene-2,4-dione (Table 1, entry 6) ${ }^{1}$ and 1-phenyl-7octene-1,3-dione (Table 1, entry 8$)^{2}$ were prepared according to published procedures. All other reagents were purchased from major chemical suppliers and were used as received.

\section{$\zeta$-Alkenyl $\beta$-diketones}

5,5-Dimethyl-8-nonene-2,4-dione (4). Dione 4 was synthesized in two steps in 36\% overall yield from methyl isobutyrate as outlined in Scheme S1.

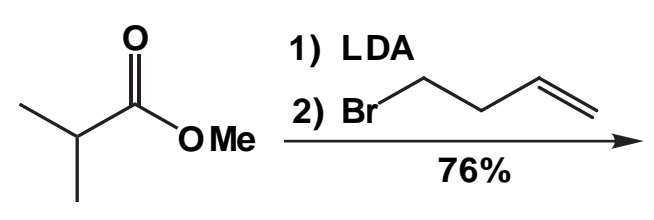

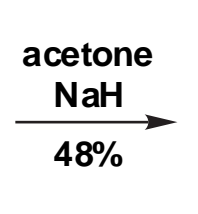

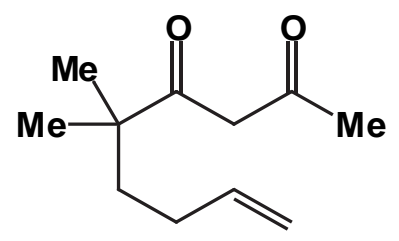

\section{Scheme S1}


A solution of methyl isobutyrate $(5.0 \mathrm{~g}, 49 \mathrm{mmol})$ in THF $(15 \mathrm{~mL})$ was added slowly to a solution of LDA [generated from $n$-BuLi $(21 \mathrm{~mL}$ in hexanes, $2.5 \mathrm{M}, 52 \mathrm{mmol})$ and diisopropylamine $(5.5 \mathrm{~g}, 54$ mmol) in THF $(65 \mathrm{~mL})]$ at $-78{ }^{\circ} \mathrm{C}$ and stirred for $20 \mathrm{~min}$. The resulting solution was treated with a solution of 4-bromo-1-butene $(7.4 \mathrm{~g}, 55 \mathrm{mmol})$ in HMPA $(10 \mathrm{~mL})$ and stirred at $-78{ }^{\circ} \mathrm{C}$ for $12 \mathrm{~h}$. Aqueous $\mathrm{HCl}(1 \mathrm{~N}, 30 \mathrm{~mL})$ was added and the resulting mixture was extracted with ether $(3 \times 50 \mathrm{~mL})$. The combined organic fractions were washed with water $(4 \times 20 \mathrm{~mL})$ and saturated $\mathrm{NaHCO}_{3}(2 \times 20 \mathrm{~mL})$, dried, and concentrated. The oily residue was distilled under vacuum $\left(64^{\circ} \mathrm{C}, 50 \mathrm{~mm} \mathrm{Hg}\right)$ to give methyl 2,2-dimethyl-5-hexenoate $(5.8 \mathrm{~g}, 76 \%)$.

A suspension of methyl 2,2-dimethyl-5-hexenoate $(3.1 \mathrm{~g}, 20 \mathrm{mmol})$ and $\mathrm{NaH}(2.0 \mathrm{~g}, 83.3 \mathrm{mmol})$ in ether $(50 \mathrm{~mL})$ and $\mathrm{DMF}(30 \mathrm{~mL})$ was heated at $70{ }^{\circ} \mathrm{C}$ for $1 \mathrm{~h}$. The resulting mixture was cooled to $0{ }^{\circ} \mathrm{C}$ and water $(30 \mathrm{~mL})$ and aqueous $\mathrm{HCl}(2 \mathrm{~N}, 20 \mathrm{~mL})$ were added. The mixture was extracted with ether, and the combined ether extracts were dried and concentrated. The resulting oily residue was chromatographed $\left(\mathrm{SiO}_{2}\right.$; hexanes-ether $\left.=50: 1 \rightarrow 30: 1\right)$ to give $2(1.75 \mathrm{~g}, 48 \%)$.

For methyl 2,2-dimethyl-5-hexenoate: ${ }^{3}{ }^{1} \mathrm{H}$ NMR: $\delta 5.76(\operatorname{tdd}, \mathrm{J}=6.4,10.4,16.8 \mathrm{~Hz}, 1 \mathrm{H})$, 4.89-5.01 (m, $2 \mathrm{H}), 3.64(\mathrm{~s}, 3 \mathrm{H}), 1.93-2.00(\mathrm{~m}, 2 \mathrm{H}), 1.58-1.62(\mathrm{~m}, 2 \mathrm{H}), 1.17(\mathrm{~s}, 6 \mathrm{H}) .{ }^{13} \mathrm{C}\left\{{ }^{1} \mathrm{H}\right\} \mathrm{NMR}: \delta$ $178.4,138.6,114.6,51.8,42.2,40.0,29.5,25.3$.

For 5,5-dimethyl-8-nonene-2,4-dione (4). Enol:keto = 20:1. ${ }^{1} \mathrm{H}$ NMR: $\delta 15.8$ (br s, $1 \mathrm{H}$ ), $5.75(\mathrm{tdd}, J=6.4,10.0,16.8 \mathrm{~Hz}, 1 \mathrm{H}), 5.57(\mathrm{~s}, 1 \mathrm{H}), 4.88-5.00(\mathrm{~m}, 2 \mathrm{H}), 2.05(\mathrm{~s}, 3 \mathrm{H}), 1.89-1.96(\mathrm{~m}, 2 \mathrm{H})$, 1.55-1.60 (m, $2 \mathrm{H}), 1.12(\mathrm{~s}, 6 \mathrm{H}) .{ }^{13} \mathrm{C}\left\{{ }^{1} \mathrm{H}\right\} \mathrm{NMR}: \delta$ 199.4, 192.5, 138.8, 114.5, 96.9, 42.4, 40.0, 29.2, 25.6, 25.3. IR (neat, $\mathrm{cm}^{-1}$ ): 2973, 2940, 1650, 1600, 1473. TLC: $R_{f}=0.72$. Anal. calcd (found) for $\mathrm{C}_{11} \mathrm{H}_{18} \mathrm{O}_{2}: \mathrm{H}, 9.95$ (9.95); C, 72.49 (72.44).

6,6-Dimethyl-9-decene-3,5-dione (Table 1, entry 1). 6,6-Dimethyl-9-decene-3,5-dione was synthesized in four steps from methyl 2,2-dimethyl-5-hexenoate in $40 \%$ overall yield employing the route shown in Scheme S2. The aldol addition and Swern oxidation were performed according to a published procedure. $^{4}$ 


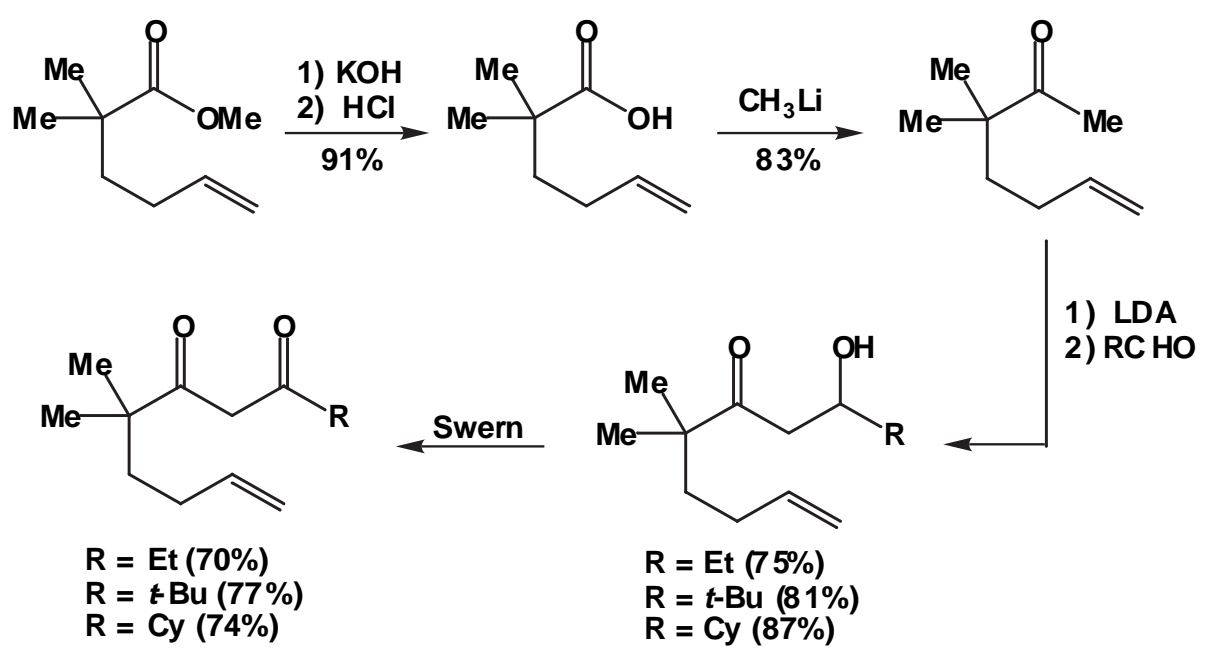

\section{Scheme S2}

A solution of methyl 2,2-dimethyl-5-hexenoate $(5.8 \mathrm{~g}, 37 \mathrm{mmol})$ and $\mathrm{KOH}(8.0 \mathrm{~g}, 143 \mathrm{mmol})$, in a water $(25 \mathrm{~mL}) / \mathrm{THF}(30 \mathrm{~mL}) / \mathrm{methanol}(80 \mathrm{~mL})$ was stirred at $25^{\circ} \mathrm{C}$ for $1 \mathrm{~h}$ and at $40{ }^{\circ} \mathrm{C}$ for $1 \mathrm{~h}$ and then treated with ice $(40 \mathrm{~g})$. The resulting mixture was acidified $(\mathrm{pH}=1)$ by slow addition of concentrated hydrochloric acid $(25 \mathrm{~mL})$ and extracted with ether $(4 \times 100 \mathrm{~mL})$. The combined organic fractions were washed with brine $(40 \mathrm{~mL})$, dried $\left(\mathrm{MgSO}_{4}\right)$, concentrated, and distilled under vacuum $\left(65^{\circ} \mathrm{C}, 200 \mathrm{mTorr}\right)$ to give 2,2-dimethyl-5-hexenoic acid (4.8 g, 91\%).

A THF solution of methyl lithium ( $97 \mathrm{~mL}, 1.4 \mathrm{M}, 136 \mathrm{mmol}$ ) was added to a solution of 2,2dimethyl-5-hexenoic acid in THF $(100 \mathrm{~mL})$ at $0{ }^{\circ} \mathrm{C}$, stirred for $3 \mathrm{~h}$, and quenched by sequential addition of $\mathrm{SiMe}_{3} \mathrm{Cl}(50 \mathrm{~mL}, 37 \mathrm{mmol})$, aqueous $\mathrm{HCl}(3 \mathrm{~N}, 20 \mathrm{~mL})$, and ice $(100 \mathrm{~g})$. The resulting mixture was extracted with ether $(3 \times 100 \mathrm{~mL})$ and the combined organic fractions were washed with brine $(50 \mathrm{~mL})$, dried $\left(\mathrm{MgSO}_{4}\right)$, concentrated, and distilled under vacuum $\left(60-65^{\circ} \mathrm{C}, 50 \mathrm{~mm} \mathrm{Hg}\right)$ to give 3,3-dimethyl-6hepten-2-one (3.6 g, 83\%).

3,3-Dimethyl-6-hepten-2-one ( $2.0 \mathrm{~g}, 11 \mathrm{mmol})$ was added slowly to a solution of LDA [generated from $n$-BuLi (3.8 mL, 2.5 M, $9.5 \mathrm{mmol})$ and diisopropylamine (1.0 g, $9.5 \mathrm{mmol})$ in THF (20 mL)] at -78 ${ }^{\circ} \mathrm{C}$ and stirred for $15 \mathrm{~min}$. Propionaldehyde $(0.55 \mathrm{~g}, 9.5 \mathrm{mmol})$ was added dropwise to the resulting white suspension, stirred for $1 \mathrm{~h}$, and quenched with saturated aqueous ammonium chloride $(6 \mathrm{~mL})$ at $-78{ }^{\circ} \mathrm{C}$. The resulting suspension was warmed to room temperature and extracted with ether $(200 \mathrm{~mL})$. The ether 
extract was washed with brine and the combined aqueous fractions were extracted with ether $(2 \times 30 \mathrm{~mL})$. The combined organic fractions were dried $\left(\mathrm{MgSO}_{4}\right)$, concentrated, and chromatographed $\left(\mathrm{SiO}_{2}\right.$; hexanes-ether $=10: 1 \rightarrow 1: 2)$ to give 3-hydroxy-6,6-dimethyl-9-decene-5-one (1.2 g, 75\%).

Solutions of DMSO (0.83 mL, $12 \mathrm{mmol})$ in $\mathrm{CH}_{2} \mathrm{Cl}_{2}(2 \mathrm{~mL})$ and 3-hydroxy-6,6-dimethyl-9decene-5-one $(1.1 \mathrm{~g}, 5.4 \mathrm{mmol})$ in $\mathrm{CH}_{2} \mathrm{Cl}_{2}(3 \mathrm{~mL})$ were added sequentially to a solution of oxalyl chloride $(0.51 \mathrm{~mL}, 5.8 \mathrm{mmol})$ in $\mathrm{CH}_{2} \mathrm{Cl}_{2}(10 \mathrm{~mL})$ at $-78^{\circ} \mathrm{C}$ and stirred for $1.5 \mathrm{~h}$ and then treated with triethylamine (5 g, $50 \mathrm{mmol}$ ). The resulting solution was stirred for $10 \mathrm{~min}$, warmed to room temperature, and treated with water $(10 \mathrm{~mL})$. Ether $(50 \mathrm{~mL})$ was added, the layers were separated, and the aqueous layer was extracted with ether $(2 \times 25 \mathrm{~mL})$. The combined organic fractions were washed with aqueous $\mathrm{HCl}(1 \mathrm{~N}, 2$ $\times 10 \mathrm{~mL})$ and saturated aqueous $\mathrm{NaHCO}_{3}(2 \times 10 \mathrm{~mL})$, dried $\left(\mathrm{MgSO}_{4}\right)$, concentrated, and chromatographed $\left(\mathrm{SiO}_{2}\right.$; hexanes-ether $\left.=50: 1 \rightarrow 25: 1\right)$ to give 6,6-dimethyl-7-decene-3,5-dione $(0.73 \mathrm{~g}$, $70 \%)$.

For 2,2-dimethyl-5-hexenoic acid: ${ }^{1}$ H NMR: $\delta 5.78(\operatorname{tdd}, J=6.3,10.2,16.5 \mathrm{~Hz}, 1 \mathrm{H}), 4.91-$ $5.04(\mathrm{~m}, 2 \mathrm{H}), 2.00-2.07(\mathrm{~m}, 2 \mathrm{H}), 1.60-1.66(\mathrm{~m}, 2 \mathrm{H}), 1.20(\mathrm{~s}, 6 \mathrm{H}) .{ }^{13} \mathrm{C}\left\{{ }^{1} \mathrm{H}\right\} \mathrm{NMR}: \delta$ 184.2, 137.6, 113.9, 41.3, 38.8, 28.5, 24.2. Anal. calcd (found) for $\mathrm{C}_{8} \mathrm{H}_{14} \mathrm{O}_{2}$ : H, 9.92 (9.81); C, 67.57 (67.42).

For 3,3-dimethyl-6-hepten-2-one: ${ }^{6}{ }^{1} \mathrm{H}$ NMR $(300 \mathrm{MHz}): \delta 5.73(\mathrm{tdd}, J=6.3,9.9,16.8 \mathrm{~Hz}, 1$ $\mathrm{H}), 4.88-4.99(\mathrm{~m}, 2 \mathrm{H}), 2.07(\mathrm{~s}, 3 \mathrm{H}), 1.84-1.96(\mathrm{~m}, 2 \mathrm{H}), 1.53-1.59(\mathrm{~m}, 2 \mathrm{H}), 1.08(\mathrm{~s}, 6 \mathrm{H}) .{ }^{13} \mathrm{C}\left\{{ }^{1} \mathrm{H}\right\}$ NMR $(75 \mathrm{MHz}): \delta 213.0,137.6,113.9,46.9,38.3,28.4,24.4,23.6$. Anal. calcd (found) for $\mathrm{C}_{9} \mathrm{H}_{16} \mathrm{O}: \mathrm{H}_{\text {, }}$ 11.50 (11.39); C, 77.09 (76.97).

For 3-hydroxy-6,6-dimethyl-9-decene-5-one. ${ }^{1} \mathrm{H}$ NMR $(300 \mathrm{MHz}): \delta$ 5.66-5.80 (m, $\left.1 \mathrm{H}\right)$, 4.89-5.00 (m, 2 H), 3.85-3.95 (m, $1 \mathrm{H}), 3.28(\mathrm{~d}, J=2.7 \mathrm{~Hz}, 1 \mathrm{H}), 2.60-2.27$ (m, $1 \mathrm{H}), 2.46(\mathrm{~m}, 1 \mathrm{H}), 1.85-$ $1.93(\mathrm{~m}, 2 \mathrm{H}), 1.36-1.63(\mathrm{~m}, 4 \mathrm{H}), 1.10(\mathrm{~s}, 6 \mathrm{H}), 0.92(\mathrm{t}, J=7.5 \mathrm{~Hz}, 3 \mathrm{H}) .{ }^{13} \mathrm{C}\left\{{ }^{1} \mathrm{H}\right\} \mathrm{NMR}(75 \mathrm{MHz}): \delta$ 216.7, 137.5, 114.0, 68.4, 46.9, 42.9, 42.3, 38.3, 28.6, 28.3, 23.5, 9.3. IR (neat, $\left.\mathrm{cm}^{-1}\right): 3445,2969,2933$, 1696. TLC: $R_{f}=0.24$. Anal. calcd (found) for $\mathrm{C}_{12} \mathrm{H}_{22} \mathrm{O}_{2}: \mathrm{H}, 11.18$ (10.92); C, 72.68 (72.51).

For 6,6-dimethyl-9-decene-3,5-dione: Enol:keto = 12:1. ${ }^{1} \mathrm{H}$ NMR: $\delta 15.8(\mathrm{~s}, 1 \mathrm{H}), 5.77$ (tdd, $J=6.4,10.0,16.8 \mathrm{~Hz}, 1 \mathrm{H}), 5.57(\mathrm{~s}, 1 \mathrm{H}), 4.90-5.01(\mathrm{~m}, 2 \mathrm{H}), 3.34$ (q, $J=7.6 \mathrm{~Hz}, 2 \mathrm{H}), 1.91-1.97$ (m, 2 $\mathrm{H}), 1.57-1.62(\mathrm{~m}, 2 \mathrm{H}), 1.14(\mathrm{~s}, 6 \mathrm{H}), 1.13(\mathrm{t}, J=7.6 \mathrm{~Hz}, 3 \mathrm{H}) .{ }^{13} \mathrm{C}\left\{{ }^{1} \mathrm{H}\right\} \mathrm{NMR}: \delta 198.9,196.9,138.8$, 
114.5, 95.6, 42.3, 40.0, 32.2, 29.3, 25.4, 9.9. IR (neat, $\left.\mathrm{cm}^{-1}\right): 2974,2939,1600,1460$. TLC: $R_{f}=0.74$. Anal. calcd (found) for $\mathrm{C}_{12} \mathrm{H}_{20} \mathrm{O}_{2}: \mathrm{H}, 10.27$ (10.13); C, 73.43 (73.21).

2,6,6-Trimethyl-9-decene-3,5-dione (Table 1, entry 2). 2,6-Dimethylheptane-3,5-dione, a solution of $n$-BuLi in hexanes (2.5 M, $5.1 \mathrm{~mL}, 12.8 \mathrm{mmol})$, and 4-bromo-1-butene (3.2 g, $24 \mathrm{mmol})$ were added sequentially to a suspension of $\mathrm{NaH}$ in $\mathrm{THF}$ at $0{ }^{\circ} \mathrm{C}$ and stirred overnight. Saturated aqueous $\mathrm{NH}_{4} \mathrm{Cl}$ and aqueous $\mathrm{HCl}(2 \mathrm{~N}, 10 \mathrm{~mL})$ were added and the resulting mixture was extracted with ether $(3 \times$ $30 \mathrm{~mL})$. The combined ether extracts were dried $\left(\mathrm{MgSO}_{4}\right)$ and distilled under vacuum $\left(50{ }^{\circ} \mathrm{C}, 300 \mathrm{~mm}\right.$ $\mathrm{Hg}$ ) to give 2,6,6-trimethyl-9-decene-3,5-dione (1.34 g, 50\%). Enol:keto = 10:1. ${ }^{1} \mathrm{H}$ NMR: $\delta 15.9$ (br s, $1 \mathrm{H}), 5.77$ (tdd, $J=6.4,10.0,16.8 \mathrm{~Hz}, 1 \mathrm{H}), 5.59(\mathrm{~s}, 1 \mathrm{H}), 4.90-5.01(\mathrm{~m}, 2 \mathrm{H}), 2.48$ (septet, $J=6.8 \mathrm{~Hz}, 1 \mathrm{H}$ ), 1.92-1.98 (m, $2 \mathrm{H}), 1.57-1.62(\mathrm{~m}, 2 \mathrm{H}), 1.14(\mathrm{~d}, J=6.8 \mathrm{~Hz}, 6 \mathrm{H}), 1.4(\mathrm{~s}, 6 \mathrm{H}) .{ }^{13} \mathrm{C}\left\{{ }^{1} \mathrm{H}\right\} \mathrm{NMR}: \delta 200.0$, 199.8, 138.8, 114.5, 94.2, 42.5, 40.1, 37.3, 25.4, 19.6. IR (neat, $\mathrm{cm}^{-1}$ ): 2968, 2872, 1594, 1466. TLC: $R_{f}$ $=0.75$. Anal. calcd (found) for $\mathrm{C}_{13} \mathrm{H}_{22} \mathrm{O}_{2}: \mathrm{H}, 10.54$ (10.46); $\mathrm{C}, 74.24$ (74.21).

2,2,6,6-Tetramethyl-9-decene-3,5-dione (Table 1, entry 3). 2,2,6,6-Tetramethyl-9-decene-3,5dione was synthesized in two steps from 3,3-dimethyl-6-hepten-2-one in 62\% overall yield employing a procedure simiar to that described above for the synthesis of 6,6-dimethyl-9-decene-3,5-dione depicted in Scheme S2.

For 3-hydroxy-2,2,6,6-tetramethyl-9-decene-5-one: ${ }^{1} \mathrm{H}$ NMR: $\delta 5.79$ (tdd, $J=6.8,10.016 .8$ $\mathrm{Hz}, 1 \mathrm{H}), 4.91-5.02(\mathrm{~m}, 2 \mathrm{H}), 3.64(\mathrm{dd}, J=1.2,10.0 \mathrm{~Hz}, 1 \mathrm{H}), 3.16(\mathrm{br} \mathrm{s}, 1 \mathrm{H}), 2.69$ (dd, $J=1.6,17.2 \mathrm{~Hz}, 1$ H), $3.38(\mathrm{dd}, J=2.0,17.2 \mathrm{~Hz}, 1 \mathrm{H}), 1.84-2.00(\mathrm{~m}, 2 \mathrm{H}), 1.58-1.62(\mathrm{~m}, 2 \mathrm{H}), 1.13(\mathrm{~s}, 3 \mathrm{H}), 1.12(\mathrm{~s}, 3 \mathrm{H})$, 0.90 (s, 9 H). ${ }^{13} \mathrm{C}\left\{{ }^{1} \mathrm{H}\right\}$ NMR: $\delta \quad 218.1,138.4,114.9,75.2,48.1,39.3,38.5,34.4,29.2,25.9,24.4$. IR (neat, $\mathrm{cm}^{-1}$ ): 3545, 2960, 2871, 1697, 1472. TLC: $R_{f}=0.45$. Anal. calcd (found) for $\mathrm{C}_{14} \mathrm{H}_{26} \mathrm{O}_{2}: \mathrm{H}$, 11.58 (11.49); C, 74.29 (74.37).

For 2,2,6,6-tetramethyl-9-decene-3,5-dione: Enol:keto $\geq 30: 1$. ${ }^{1} \mathrm{H}$ NMR: $\delta 16.2$ (s, $\left.1 \mathrm{H}\right), 5.77$ (tdd, $J=6.4,10.0,17.2 \mathrm{~Hz}, 1 \mathrm{H}), 5.71(\mathrm{~s}, 1 \mathrm{H}), 4.8-5.08$ (m, $2 \mathrm{H}), 1.92-1.99(\mathrm{~m}, 2 \mathrm{H}), 1.58-1.62(\mathrm{~m}, 2 \mathrm{H})$, 1.17 (s, 9 H), 1.15 (s, $6 \mathrm{H}) .{ }^{13} \mathrm{C}\left\{{ }^{1} \mathrm{H}\right\}$ NMR: $\delta$ 201.6, 200.5, 138.9, 114.5, 91.9, 42.8, 40.1, 39.6, 29.3, 27.6, 25.5. IR (neat, $\mathrm{cm}^{-1}$ ): 2970, 2942, 1601, 1470. TLC: $R_{f}=0.87$. Anal. calcd (found) for $\mathrm{C}_{14} \mathrm{H}_{24} \mathrm{O}_{2}: \mathrm{H}, 10.78$ (10.69); C, 74.95 (75.01). 
1-Cyclohexyl-4,4-dimethyl-7-octene-1,3-dione (Table 1, entry 4). 1-Cyclohexyl-4,4dimethyl-7-octene-1,3-dione was synthesized in two steps from 3,3-dimethyl-6-hepten-2-one in 64\% overall yield employing a procedure similar to that described above for the synthesis of 6,6-dimethyl-9decene-3,5-dione depicted in Scheme S2.

For 1-Cyclohexyl-1-hydroxy-4,4-dimethyl-7-octene-3-one: ${ }^{1} \mathrm{H}$ NMR $(300 \mathrm{MHz}): \delta 5.71$ (tdd, $J=6.3,10.2,16.2 \mathrm{~Hz}, 1 \mathrm{H}), 4.87-4.98(\mathrm{~m}, 2 \mathrm{H}), 3.71$ (br s, $1 \mathrm{H}), 2.40-2.49$ (m, $1 \mathrm{H}), 2.60-2.66$ (m, 1 H), 1.83-1.90 (m, $2 \mathrm{H}), 1.69-1.75(\mathrm{~m}, 2 \mathrm{H}), 1.53-1.63(\mathrm{~m}, 4 \mathrm{H}), 0.94-1.30(\mathrm{~m}, 7 \mathrm{H}), 1.08$ (s, $6 \mathrm{H})$. ${ }^{13} \mathrm{C}\left\{{ }^{1} \mathrm{H}\right\}$ NMR: $\delta 217.0,137.5,114.0,71.0,47.0,42.2,39.8,38.3,28.3,27.6,25.8,25.5,25.4,23.5$. IR (neat, $\mathrm{cm}^{-1}$ ): $3507,2925,2852,1696,1641$. TLC: $R_{f}=0.31$. Anal. calcd (found) for $\mathrm{C}_{11} \mathrm{H}_{16} \mathrm{O}_{2}: \mathrm{H}_{\text {, }}$ 11.18 (11.12); C, 76.14 (76.07).

For 1-Cyclohexyl-4,4-dimethyl-7-octene-1,3-dione: Enol:keto = 20:1. ${ }^{1} \mathrm{H}$ NMR: $\delta$ 16.0 (s, 1 H), $5.77(\mathrm{tdd}, J=6.4,10.0,15.2 \mathrm{~Hz}, 1 \mathrm{H}), 5.58(\mathrm{~s}, 1 \mathrm{H}), 4.98(\mathrm{qd}, J=1.6,17.2 \mathrm{~Hz}, 1 \mathrm{H}), 4.91(\mathrm{tdd}, J=1.2$, 2.0, 10.4 Hz, $1 \mathrm{H}), 2.19$ (tt, $J=3.2,11.6 \mathrm{~Hz}, 1 \mathrm{H}), 1.91-1.98$ (m, $2 \mathrm{H}), 1.78-1.87$ (m, $4 \mathrm{H}), 1.66-1.70$ (m, 1 $\mathrm{H}), 1.57-1.60(\mathrm{~m}, 2 \mathrm{H}), 1.18-1.43(\mathrm{~m}, 5 \mathrm{H}), 1.14(\mathrm{~s}, 6 \mathrm{H}) .{ }^{13} \mathrm{C}\left\{{ }^{1} \mathrm{H}\right\} \mathrm{NMR}: \delta 200.4,198.6,138.9,114.5$, 94.5, 47.2, 42.6, 40.1, 29.8, 29.3, 26.0, 25.4. IR (neat, $\left.\mathrm{cm}^{-1}\right): 2930,2855,1620,1597,1450$. TLC: $R_{f}=$ 0.50. Anal. calcd (found) for $\mathrm{C}_{16} \mathrm{H}_{26} \mathrm{O}_{2}$ : H, 10.47 (10.58); C, 76.75 (76.59).

6-Methyl-9-decene-3,5-dione (Table 1, entry 5). 6-Methyl-9-decene-3,5-dione was synthesized from 3,5-heptadione and 4-bromo-1-butene in $33 \%$ yield after vacuum distillation $\left(70{ }^{\circ} \mathrm{C}, 50 \mathrm{mmHg}\right)$ employing a procedure similiar to that used to synthesize 2,6,6-trimethyl-9-decene-3,5-dione. Enol:keto= 5:1. ${ }^{1} \mathrm{H}$ NMR: $\delta 15.5$ (br s, $\left.1 \mathrm{H}\right), 5.72-5.82(\mathrm{~m}, 1 \mathrm{H}), 5.47(\mathrm{~s}, 1 \mathrm{H}), 4.94-5.03(\mathrm{~m}, 2 \mathrm{H}), 2.32(\mathrm{q}, J=7.6$ $\mathrm{Hz}, 2 \mathrm{H}), 2.04(\mathrm{q}, J=7.6 \mathrm{~Hz}, 2 \mathrm{H}), 1.69-1.78(\mathrm{~m}, 1 \mathrm{H}), 1.41-1.51(\mathrm{~m}, 1 \mathrm{H}), 1.05-1.15(\mathrm{~m}, 7 \mathrm{H}) .{ }^{13} \mathrm{C}\left\{{ }^{1} \mathrm{H}\right\}$ NMR: $\delta$ 197.3, 196.5, 138.3, 115.1, 97.7, 41.7, 33.3, 31.9, 31.6, 17.7, 9.8. IR (neat, $\mathrm{cm}^{-1}$ ): 2975, 2935, 1603, 1454, 911. TLC: $R_{f}=0.78$. Anal. calcd (found) for $\mathrm{C}_{11} \mathrm{H}_{18} \mathrm{O}_{2}: \mathrm{H}, 9.95$ (10.03); C, 72.49 (72.38).

2,2-Dimethyl-9-decene-3,5-dione (Table 1, entry 7). Enol:keto = 19:1. 2,2-Dimethyl-9decene-3,5-dione was synthesized from 5,5-dimethyl-hexane-2,4-dione and 4-bromo-1-butene in $27 \%$ yield following vacuum distillation $\left(58^{\circ} \mathrm{C}, 250 \mathrm{mTorr}\right)$ employing a procedure similar to that used to synthesize 6-methyl-9-decene-3,5-dione. ${ }^{1} \mathrm{H}$ NMR: $\delta 15.9$ (br s, $\left.1 \mathrm{H}\right), 5.78(\mathrm{tdd}, J=6.8,10.2,16.8 \mathrm{~Hz}, 1 \mathrm{H}), 5.58$ (s, 
$1 \mathrm{H}), 4.97-5.05(\mathrm{~m}, 2 \mathrm{H}), 2.31(\mathrm{t}, J=7.6 \mathrm{~Hz}, 2 \mathrm{H}), 2.09$ (q, $J=7.6 \mathrm{~Hz}, 2 \mathrm{H}), 1.71$ (quintet, $J=7.6 \mathrm{~Hz}, 2 \mathrm{H}$ ), $1.16(\mathrm{~s}, 9 \mathrm{H}) .{ }^{13} \mathrm{C}\left\{{ }^{1} \mathrm{H}\right\} \mathrm{NMR}: \delta 200.5,195.4,138.1,115.5,95.4,39.2,38.2,33.3,27.5,25.0$. IR (neat, $\mathrm{cm}^{-1}$ ): 2976, 2935, 1604, 1455. TLC: $R_{f}=0.83$. Anal. calcd (found) for $\mathrm{C}_{12} \mathrm{H}_{20} \mathrm{O}_{2}: \mathrm{H}, 10.27$ (10.16); C, 73.43 (73.28).

7,7-Dimethyl-8-nonene-2,4-dione (Table 1, entry 9). 7,7-Dimethyl-8-nonene-2,4-dione was synthesized from (2-methyl-[1,3]dioxolan-2-yl)acetaldehyde in 29\% overall yield employing the procedure shown in Scheme S3.
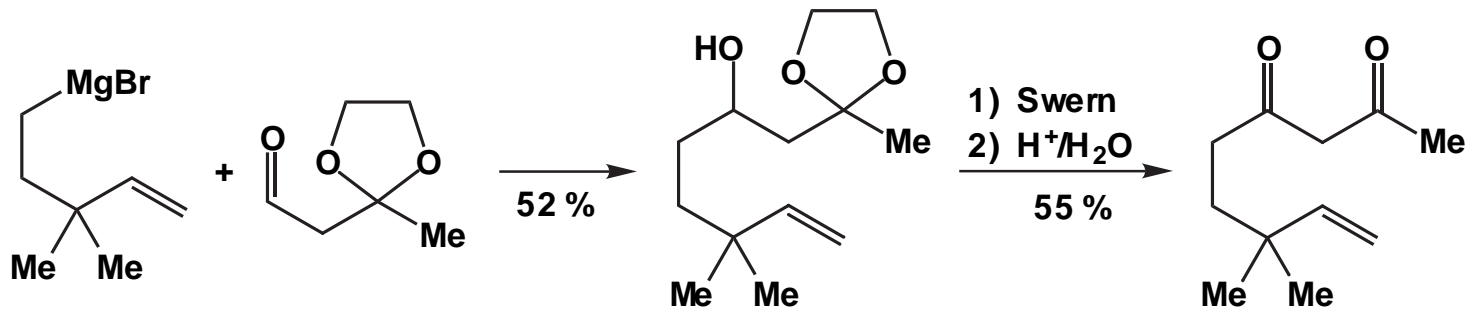

\section{Scheme S3}

5-Bromo-3,3-dimethyl-1-pentene $(0.73 \mathrm{~g}, 4.5 \mathrm{mmol})$ was added dropwise to a suspension of $\mathrm{Mg}$ $(0.24 \mathrm{~g}, 10.0 \mathrm{mmol})$ in ether $(25 \mathrm{~mL})$ and the mixture was refluxed for $20 \mathrm{~min}$ and cooled to room temperature. (2-Methyl-[1,3]dioxolan-2-yl)acetaldehyde $(0.52 \mathrm{~g}, 4.0 \mathrm{mmol})$ was added dropwise and the resulting suspension was stirred at room temperature for $5 \mathrm{~h}$, quenched with saturated aqueous $\mathrm{NH}_{4} \mathrm{Cl}$, and extracted with ether. The combined organic fractions were washed with brine, dried $\left(\mathrm{MgSO}_{4}\right)$, and concentrated under vacuum. The resulting oily residue was chromatographed $\left(\mathrm{SiO}_{2}\right.$; hexanes-ether $=25: 1$ $\rightarrow$ 5:1) to give 5,5-dimethyl-1-(2-methyl-[1,3]dioxolan-2-yl)-hept-6-en-2-ol (0.47 g, 52\%).

5,5-Dimethyl-1-(2-methyl-[1,3]dioxolan-2-yl)-hept-6-en-2-ol (1.26 g, $5.50 \mathrm{mmol})$ was oxidized employing a procedure analogous to that used to synthesize 6,6-dimethyl-9-decene-3,5-dione and was purified by chromatography $\left(\mathrm{SiO}_{2} ;\right.$ hexanes-ether $\left.=25: 1\right)$ to give 7,7-dimethyl-8-nonene-2,4-dione $(0.55$ $\mathrm{g}, 55 \%)$ as a yellow oil.

For 5,5-Dimethyl-1-(2-methyl-[1,3]dioxolan-2-yl)-6-hepten-2-ol: ${ }^{1} \mathrm{H}$ NMR: $\delta \quad 5.78-5.71$ (m, $1 \mathrm{H}), 4.91(\mathrm{~s}, 1 \mathrm{H}), 4.87(\mathrm{dd}, J=1.6,6.8 \mathrm{~Hz}, 1 \mathrm{H}), 3.98(\mathrm{~m}, 4 \mathrm{H}), 3.84-3.78(\mathrm{~m}, 1 \mathrm{H}), 3.59(\mathrm{~s}, 1 \mathrm{H})$, 
$1.82(\mathrm{dd}, J=1.6,14.8 \mathrm{~Hz}, 1 \mathrm{H}), 1.74(\mathrm{dd}, J=9.6,14.8 \mathrm{~Hz}, 1 \mathrm{H}), 1.50-1.37(\mathrm{~m}, 2 \mathrm{H}), 1.35$ (s, $3 \mathrm{H}), 1.34-$ $1.22(\mathrm{~m}, 2 \mathrm{H}), 0.98(\mathrm{~s}, 6 \mathrm{H}) .{ }^{13} \mathrm{C}\left\{{ }^{1} \mathrm{H}\right\}$ NMR: $\delta$ 148.6, 110.8, 68.9, 65.0, 64.6, 45.2, 38.5, 36.6, 32.7, 27.1, 26.9, 24.5. IR (neat, $\mathrm{cm}^{-1}$ ): 3526, 3079, 2955, 2884, 1645, 1557, 1540, 1472, 1456, 1416, 1376, 1302, 1105, 1039. TLC: $R_{f}=0.19$. Anal. calcd (found) for $\mathrm{C}_{13} \mathrm{H}_{24} \mathrm{O}_{3}: \mathrm{H}, 10.59$ (10.47); C, 68.38 (68.19).

For 7,7-Dimethyl-8-nonene-2,4-dione: Enol:keto $=10: 1 .{ }^{1} \mathrm{H}$ NMR: $\delta 15.46(\mathrm{~s}, 1 \mathrm{H}), 5.72$ (dd, $J=10.8,17.4 \mathrm{~Hz}, 1 \mathrm{H}), 5.47$ (s, $1 \mathrm{H}), 4.96(\mathrm{dd}, J=1.6,10.8 \mathrm{~Hz}, 1 \mathrm{H}), 4.92(\mathrm{dd}, J=1.6,17.4 \mathrm{~Hz}, 1 \mathrm{H})$, 2.22-2.16 (m, 2 H), 2.03 (s, 3 H), 1.61-1.57 (m, 2 H), 1.00 (s, 6 H). ${ }^{13} \mathrm{C}\left\{{ }^{1} \mathrm{H}\right\}$ NMR: $\delta$ 195.7, 191.0, 147.6, 111.7, 100.0, 38.2, 36.7, 34.5, 26.9, 25.1. IR (neat, $\mathrm{cm}^{-1}$ ): 3082, 2997, 2961, 2928, 2869, 1728, $1711,1620,1454,1416,1362,1285,1239,1160,1127,1001$. TLC: $R_{f}=0.48$. Anal. calcd (found) for $\mathrm{C}_{11} \mathrm{H}_{18} \mathrm{O}_{2}: \mathrm{H}, 9.95$ (10.02); C, 72.49 (72.36).

1-(1-But-3-enylcyclohexyl)butane-1,3-dione (Table 1, entry 10). 1-(1-But-3enylcyclohexyl)butane-1,3-dione was synthesized in five steps in $19 \%$ overall yield from methyl cyclohexanecarboxylate employing the route shown in Scheme S4.

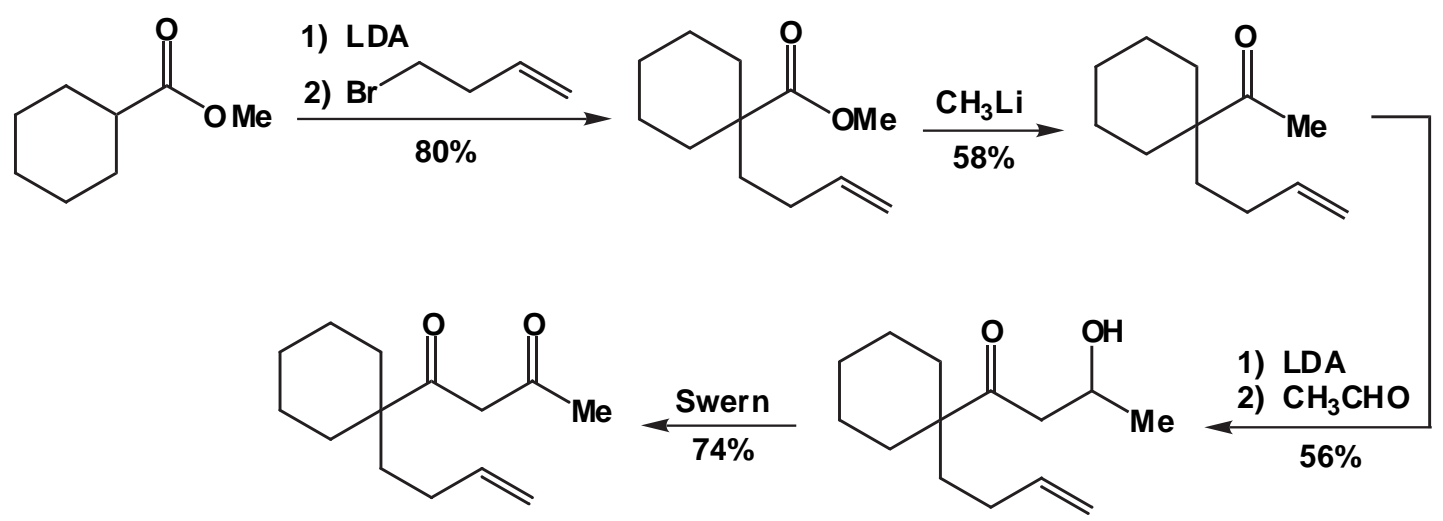

\section{Scheme S4}

Methyl cyclohexanecarboxylate $(8.0 \mathrm{~g}, 56.3 \mathrm{mmol})$ was added to a solution of LDA [generated from $n$-BuLi (2.5 N in hexanes, $22.5 \mathrm{~mL}, 56.3 \mathrm{mmol})$ and diisopropylamine $(7.0 \mathrm{~g}, 69 \mathrm{mmol})$ in THF (60 $\mathrm{mL}$ ) at $0^{\circ} \mathrm{C}$ ] over $1 \mathrm{~h}$ at $-78{ }^{\circ} \mathrm{C}$ and the resulting solution was stirred for $30 \mathrm{~min}$. A solution of 4-bromo1-butene $(8.0 \mathrm{~g}, 60 \mathrm{mmol})$ in HMPA $(10 \mathrm{~mL})$ was added dropwise over $30 \mathrm{~min}$ at $-78{ }^{\circ} \mathrm{C}$ and stirred for 40 min, warmed to room temperature, and stirred for $2 \mathrm{~h}$. Ice $(20 \mathrm{~g})$ and aqueous $\mathrm{HCl}(3 \mathrm{~N}, 30 \mathrm{~mL})$ were 
added and the resulting mixture was extracted with ether $(4 \times 50 \mathrm{~mL})$. The combined organic fractions were washed with water $(3 \times 10 \mathrm{~mL})$, dried $\left(\mathrm{MgSO}_{4}\right)$, concentrated, and distilled under vacuum $\left(64-66^{\circ} \mathrm{C}\right.$, 350 mTorr) to give 1-(3-butenyl)-1-carbomethoxycyclohexane (8.8 g, 80\%).

A solution of methyl lithium $(50 \mathrm{~mL}, 1.4 \mathrm{~N}$ in THF, $70 \mathrm{mmol})$ was added to a solution of 1-(3butenyl)-1-carbomethoxycyclohexane $(6.0 \mathrm{~g}, 30 \mathrm{mmol})$ in $\mathrm{THF}(40 \mathrm{~mL})$ at $0{ }^{\circ} \mathrm{C}$ and stirred for $1 \mathrm{~h}$. Ice $(20 \mathrm{~g})$ and aqueous $\mathrm{HCl}(3 \mathrm{~N}, 20 \mathrm{~mL})$ were added and the mixture was extracted with ether $(3 \times 50 \mathrm{~mL})$. The combined organic fractions were dried $\left(\mathrm{MgSO}_{4}\right)$, concentrated, and chromatographed $\left(\mathrm{SiO}_{2}\right.$; hexanes-EtOAc = 50:1) to give 1-acetyl-1-(3-butenyl)cyclohexane (3.2 g, 58\%).

1-Acetyl-1-(3-butenyl)cyclohexane $(2.0 \mathrm{~g}, 11 \mathrm{mmol})$ was added slowly to a solution of LDA [generated from $n$-BuLi $(5.5 \mathrm{~mL}, 2.5 \mathrm{M}, 14 \mathrm{mmol})$ and diisopropylamine (1.4 g, $14 \mathrm{mmol})$ in THF (20 $\mathrm{mL})]$ at $-78{ }^{\circ} \mathrm{C}$ and stirred for $15 \mathrm{~min}$. Acetaldehyde $(0.54 \mathrm{~g}, 12 \mathrm{mmol})$ was added dropwise to the resulting white suspension and stirred for $1 \mathrm{~h}$. Aqueous ammonium chloride $(6 \mathrm{~mL})$ was added at $-78^{\circ} \mathrm{C}$ and the resulting mixture was extracted with ether $(200 \mathrm{~mL})$. The ether extract was washed with brine and the combined aqueous fractions were extracted with ether $(2 \times 30 \mathrm{~mL})$. The combined organic fractions were dried $\left(\mathrm{MgSO}_{4}\right)$, concentrated, and chromatographed $\left(\mathrm{SiO}_{2}\right.$; hexanes-ether $\left.=33: 1 \rightarrow 1: 2\right)$ to give 1 (1-but-3-enylcyclohexyl)-3-hydroxybutan-1-one (1.4 g, 56\%).

(1-But-3-enylcyclohexyl)-3-hydroxybutan-1-one was oxidized employing the procedure used to synthesize 6,6-dimethyl-9-decene-3,5-dion and was purified by chromatography $\left(\mathrm{SiO}_{2}\right.$; hexanes-ether $=$ $50: 1 \rightarrow 25: 1)$ to give 1-(1-but-3-enyl-cyclohexyl)-butane-1,3-dione in 74\% yield.

For 1-(3-butenyl)-1-carbomethoxycyclohexane. ${ }^{1} \mathrm{H}$ NMR $(300 \mathrm{MHz}): \delta 5.83(\mathrm{tdd}, J=6.3$, 10.5, 16.8 Hz, $1 \mathrm{H}), 4.99-5.10$ (m, $2 \mathrm{H}), 3.76$ (s, $3 \mathrm{H}), 2.17$ (m, $2 \mathrm{H}), 1.99-2.06$ (m, $2 \mathrm{H}), 1.62-1.78$ (m, 4 $\mathrm{H}), 1.25-1.47(\mathrm{~m}, 6 \mathrm{H}) .{ }^{13} \mathrm{C}\left\{{ }^{1} \mathrm{H}\right\} \mathrm{NMR}: \delta \quad 176.4,137.8,117.7,50.7,46.1,39.0,33.5,27.8,25.2,22.5$. TLC: $R_{f}=0.66$. Anal. calcd (found) for $\mathrm{C}_{12} \mathrm{H}_{20} \mathrm{O}_{2}: \mathrm{H}, 10.27$ (10.27); C, 73.43 (73.33).

For 1-Acetyl-1-(3-butenyl)cyclohexane: ${ }^{1} \mathrm{H}$ NMR $(300 \mathrm{MHz}): \delta 5.83(\mathrm{tdd}, J=6.6,10.2,16.8$ Hz, $1 \mathrm{H})$, 5.00-5.10 (m, 2 H), 2.19 (s, 3 H), 2.06-2.12 (m, 2 H), 1.90-1,98 (m, 2 H), 1.61-1.68 (m, 6 H), 1.36-1.39 (m, $4 \mathrm{H}) .{ }^{13} \mathrm{C}\left\{{ }^{1} \mathrm{H}\right\} \mathrm{NMR}(75 \mathrm{MHz}): \delta 212.8,137.6,113.9,51.2,37.4,32.6,27.4,25.4,24.6$, 22.2. TLC: $R_{f}=0.55$. Anal. calcd (found) for $\mathrm{C}_{12} \mathrm{H}_{20} \mathrm{O}: \mathrm{H}, 11.18$ (11.27); C, 79.94 (79.71). 
For 1-(1-But-3-enylcyclohexyl)-3-hydroxy-1-butanone: ${ }^{1} \mathrm{H}$ NMR $(300 \mathrm{MHz}): \delta 5.71(\operatorname{tdd}, J$ $=6.3,10.2,16.8 \mathrm{~Hz}, 1 \mathrm{H}), 4.89-4.99(\mathrm{~m}, 2 \mathrm{H}), 4.12-4.20(\mathrm{~m}, 1 \mathrm{H}), 3.43(\mathrm{br} \mathrm{s}, 1 \mathrm{H}), 2.64(\mathrm{dd}, J=2.7,18.0$ Hz, 1 H), 2.43 (dd, $J=9.0,18.0$ Hz, 1 H), 1.94-1.99 (m, 2 H), 1.77-1.86 (m, 2 H), 1.50-1.57 (m, 5 H), 1.23 (br s, $6 \mathrm{H}), 1.17(\mathrm{~d}, J=6.3 \mathrm{~Hz}, 3 \mathrm{H}) .{ }^{13} \mathrm{C}\left\{{ }^{1} \mathrm{H}\right\} \operatorname{NMR}(75 \mathrm{MHz}): \delta \quad 216.5,137.4,114.1,63.3,51.2,44.2$, 37.3, 32.3, 27.4, 25.2, 22.1, 21.7. IR (neat, $\mathrm{cm}^{-1}$ ): 3439, 2931, 2860, 1693. TLC: $R_{f}=0.17$. Anal. calcd (found) for $\mathrm{C}_{14} \mathrm{H}_{24} \mathrm{O}_{2}$ : $\mathrm{H}, 10.78$ (10.78); C, 74.95 (74.91).

For 1-(1-But-3-enyl-cyclohexyl)-1,3-butanedione: Enol:keto = 10:1. ${ }^{1} \mathrm{H}$ NMR: $\delta 16.0$ (br s, $1 \mathrm{H}), 5.73$ (tdd, $J=6.4,10.0,16.8 \mathrm{~Hz}, 1 \mathrm{H}), 5.62(\mathrm{~s}, 1 \mathrm{H}), 4.87-4.98$ (m, $2 \mathrm{H}), 2.07$ (s, $3 \mathrm{H}), 1.86-1.97$ (m, $4 \mathrm{H}), 1.47-1.61(\mathrm{~m}, 5 \mathrm{H}), 1.26-1.42(\mathrm{~m}, 5 \mathrm{H}) .{ }^{13} \mathrm{C}\left\{{ }^{1} \mathrm{H}\right\} \mathrm{NMR}: \delta \quad 200.0,191.1,138.9,114.5,98.1,46.8$, 39.8, 33.8, 28.3, 26.3, 25.3, 22.9. IR (neat, $\mathrm{cm}^{-1}$ ): 2933, 2855, 2359, 2340, 1601, 1455. TLC: $R_{f}=0.69$. Anal. calcd (found) for $\mathrm{C}_{14} \mathrm{H}_{24} \mathrm{O}_{2}$ : $\mathrm{H}, 9.97$ (9.81); C, 75.63 (75.46).

\section{Cyclohexenones}

2-Acetyl-3,6,6-trimethyl-2-cyclohexenone (5) (Catalytic in 2). Alkenyl dione 4 (91 mg, 0.50 $\mathrm{mmol}$ ) was added via syringe pump over $3 \mathrm{~h}$ into a well-stirred suspension of $\mathrm{CuCl}_{2}$ (200 $\left.\mathrm{mg}, 1.48 \mathrm{mmol}\right)$ and $2(7 \mathrm{mg}, 0.027 \mathrm{mmol})$ in 1,2-dichloroethane (DCE, $10 \mathrm{~mL})$. The resulting mixture was filtered though a plug of silica gel and eluted with ether $(30 \mathrm{~mL})$. The resulting solution was dried $\left(\mathrm{MgSO}_{4}\right)$ and concentrated to give an oil that was chromatographed $\left(\mathrm{SiO}_{2}\right.$, hexane-ether $\left.=5: 1 \rightarrow 1: 2\right)$ to give $\mathbf{5}(86 \mathrm{mg}$, $96 \%$.

5 (Catalytic in 2 and $\left.\mathbf{C u C l}_{2}\right)$. Alkenyl dione 4 (91 $\mathrm{mg}, 0.50 \mathrm{mmol}$ ) was added slowly over $2 \mathrm{~h}$ to a suspension of $2(7 \mathrm{mg}, 0.027 \mathrm{mmol}), \mathrm{CuCl}_{2}(8 \mathrm{mg}, 0.06 \mathrm{mmol})$, and $\mathrm{HCl}\left(2 \mathrm{~N}\right.$ in $\mathrm{Et}_{2} \mathrm{O}, 40 \mu \mathrm{l}, 0.08$ $\mathrm{mmol})$ in DCE $(10 \mathrm{~mL})$ under $\mathrm{O}_{2}(1 \mathrm{~atm})$. Work-up and chromatography gave 5 (64 mg, 71\%).

For 5: ${ }^{1} \mathrm{H}$ NMR: $\delta$ 2.34-2.37 (m, $\left.2 \mathrm{H}\right), 2.25(\mathrm{~s}, 3 \mathrm{H}), 1.87(\mathrm{~s}, 3 \mathrm{H}), 1.78(\mathrm{t}, J=6.4 \mathrm{~Hz}, 2 \mathrm{H}), 1.08$ (s, $6 \mathrm{H}) .{ }^{13} \mathrm{C}\left\{{ }^{1} \mathrm{H}\right\}$ NMR: $\delta$ 205.0, 202.1, 157.7, 138.2, 40.5, 35.5, 31.6, 29.5, 24.1, 21.6. IR (neat, $\mathrm{cm}^{-1}$ ): 2965, 2924, 2701, 1662, 1626. TLC: $R_{f}=0.22$. Anal. calcd (found) for $\mathrm{C}_{11} \mathrm{H}_{16} \mathrm{O}_{2}: \mathrm{H}, 8.95$ (9.01); C, 73.30 (73.16). 
The remaining cyclohexenones were synthesized employing a procedure analogous to that used to synthesize $\mathbf{5}$ employing a catalytic amount of $\mathbf{2}$ and a stoichiometric amount of $\mathrm{CuCl}_{2}$.

3,6,6-Trimethyl-2-propionyl-2-cyclohexenone (Table 1, entry 1). ${ }^{1} \mathrm{H}$ NMR: $\delta 2.51(\mathrm{q}, J=$ $7.2 \mathrm{~Hz}, 2 \mathrm{H}), 2.34$ (t, $J=6.0 \mathrm{~Hz}, 2 \mathrm{H}), 1.82(\mathrm{~s}, 3 \mathrm{H}), 1.77$ (t, $J=6.0 \mathrm{~Hz}, 2 \mathrm{H}), 1.07$ (s, $6 \mathrm{H}), 1.03(\mathrm{t}, J=7.6$ $\mathrm{Hz}, 3 \mathrm{H}) .{ }^{13} \mathrm{C}\left\{{ }^{1} \mathrm{H}\right\} \mathrm{NMR}: \delta$ 208.2, 202.2, 157.0, 138.1, 40.5, 37.2, 35.4, 29.3, 24.1, 21.5, 7.9. IR (neat, $\mathrm{cm}^{-1}$ ): $2975,2926,1704,1661$. TLC: $R_{f}=0.22$. Anal. calcd (found) for $\mathrm{C}_{12} \mathrm{H}_{18} \mathrm{O}_{2}: \mathrm{H}, 9.34$ (9.36); C, 74.19 (73.99).

2-Isobutyryl-3,6,6-trimethyl-2-cyclohexenone (Table 1, entry 2). ${ }^{1} \mathrm{H}$ NMR : $\delta 2.79$ (septet, $J=6.8 \mathrm{~Hz}, 1 \mathrm{H}), 2.33-2.36(\mathrm{~m}, 2 \mathrm{H}), 1.81(\mathrm{~s}, 3 \mathrm{H}), 1.77(\mathrm{t}, J=6.0 \mathrm{~Hz}, 2 \mathrm{H}), 1.07(\mathrm{~s}, 6 \mathrm{H}), 1.03(\mathrm{~d}, J=7.2$ Hz, 6 H). ${ }^{13} \mathrm{C}\left\{{ }^{1} \mathrm{H}\right\}$ NMR: $\delta$ 211.2, 202.4, 157.7, 137.6, 41.1, 40.5, 35.4, 29.3, 24.1, 21.8, 17.9. IR (neat, $\mathrm{cm}^{-1}$ ): 2970, 2930, 1697, 1660. TLC: $R_{f}=0.36$. Anal. calcd (found) for $\mathrm{C}_{13} \mathrm{H}_{20} \mathrm{O}_{2}: \mathrm{H}, 9.68$ (9.61); C, 74.96 (74.97).

2-(2,2-Dimethyl-propionyl)-3,6,6-trimethyl-2-cyclohexenone (Table 1, entry 3). White needles, mp 55-56 ${ }^{\circ} \mathrm{C} .{ }^{1} \mathrm{H}$ NMR: $\delta 2.32(\mathrm{t}, J=6.0 \mathrm{~Hz}, 2 \mathrm{H}), 1.78(\mathrm{t}, J=6.0 \mathrm{~Hz}, 2 \mathrm{H}), 1.77(\mathrm{~s}, 3 \mathrm{H}), 1.10$ (s, $9 \mathrm{H}), 1.07(\mathrm{~s}, 6 \mathrm{H}) .{ }^{13} \mathrm{C}\left\{{ }^{1} \mathrm{H}\right\} \mathrm{NMR}: \delta \quad 215.5,202.3,154.9,138.6,44.7,40.6,35.7,29.0,27.3,24.2$, 22.2. IR (neat, $\mathrm{cm}^{-1}$ ): $3011,2970,1740,1439,1369,1215$. TLC: $R_{f}=0.27$. Anal. calcd (found) for $\mathrm{C}_{11} \mathrm{H}_{16} \mathrm{O}_{2}: \mathrm{H}, 9.97$ (9.89); C, 75.63 (75.71).

2-Cyclohexanecarbonyl-3,6,6-trimethyl-2-cyclohexenone (Table 1, entry 4). White powder, mp 60-61 ${ }^{\circ} \mathrm{C} .{ }^{1} \mathrm{H}$ NMR: $\delta$ 2.50-2.52 (m, $\left.1 \mathrm{H}\right), 2.36(\mathrm{t}, J=6.0 \mathrm{~Hz}, 2 \mathrm{H}), 1.82-1.86(\mathrm{~m}, 2 \mathrm{H}), 1.83(\mathrm{~s}, 3 \mathrm{H})$, $1.79(\mathrm{t}, J=6.0 \mathrm{~Hz}, 2 \mathrm{H}), 1.72(\mathrm{br} \mathrm{d}, J=6.8 \mathrm{~Hz}, 2 \mathrm{H}), 1.62(\mathrm{br} \mathrm{d}, J=7.2 \mathrm{~Hz}, 1 \mathrm{H}), 1.05-1.31(\mathrm{~m}, 5 \mathrm{H}), 1.10$ (s, $6 \mathrm{H}) .{ }^{13} \mathrm{C}\left\{{ }^{1} \mathrm{H}\right\} \mathrm{NMR}: \delta \quad 210.7,202.5,157.6,137.8,50.9,40.6,35.5,29.4,28.2,24.2,21.9$. IR (neat, $\mathrm{cm}^{-1}$ ): 3024, 2971, 1729, 1375. TLC: $R_{f}=0.27$. Anal. calcd (found) for $\mathrm{C}_{16} \mathrm{H}_{24} \mathrm{O}_{2}: \mathrm{H}, 9.74$ (9.89); C, 77.38 (77.26).

6-Ethyl-3-methyl-2-propionyl-2-cyclohexenone (Table 1, entry 5). ${ }^{1} \mathrm{H}$ NMR: $\delta 2.55(\mathrm{q}, J=$ 7.2 Hz, $2 \mathrm{H}), 2.24-2.46$ (m, $3 \mathrm{H}), 1.98$ (qd, $J=4.4,13.2 \mathrm{~Hz}, 1 \mathrm{H}), 1.80$ (s, $3 \mathrm{H}), 1.80-1.86(\mathrm{~m}, 1 \mathrm{H}), 1.05$ $(\mathrm{d}, J=6.8 \mathrm{~Hz}, 3 \mathrm{H}), 1.00(\mathrm{t}, J=7.6 \mathrm{~Hz}, 3 \mathrm{H}) .{ }^{13} \mathrm{C}\left\{{ }^{1} \mathrm{H}\right\} \mathrm{NMR}: \delta \quad 208.0,199.7,158.3,139.3,40.9,37.4$, 
31.7, 30.0, 21.6, 15.1, 7.8. IR (neat, $\mathrm{cm}^{-1}$ ): 2974, 2934, 1700, 1661, 1376. TLC: $R_{f}=0.24$. Anal. calcd (found) for $\mathrm{C}_{11} \mathrm{H}_{16} \mathrm{O}_{2}$ : $\mathrm{H}, 8.95$ (9.00); C, 73.30 (73.15).

2-Acetyl-3-methyl-2-cyclohexenone (Table 1, entry 6). ${ }^{1} \mathrm{H}$ NMR: $\delta$ 2.33-2.38 (m, $4 \mathrm{H}$ ), 2.27 (s, $3 \mathrm{H}), 1.94$ (quintet, $J=6.4 \mathrm{~Hz}, 2 \mathrm{H}), 1.89$ (s, $3 \mathrm{H}) .{ }^{13} \mathrm{C}\left\{{ }^{1} \mathrm{H}\right\}$ NMR: $\delta$ 204.6, 197.1, 160.1, 139.9, 37.6, 32.4, 31.9, 21.9. IR (neat, $\mathrm{cm}^{-1}$ ): $2950,1701,1662,1425$. TLC: $R_{f}=0.16$. Anal. calcd (found) for $\mathrm{C}_{9} \mathrm{H}_{12} \mathrm{O}_{2}: \mathrm{H}, 7.95$ (7.83); C, 71.03 (70.90).

2-(2,2-Dimethylpropionyl)-3-methyl-2-cyclohexenone (Table 1, entry 7). ${ }^{1} \mathrm{H}$ NMR: $\delta 2.37$ (t, $J=6.0 \mathrm{~Hz}, 2 \mathrm{H}$ ), 2.33 (t, $J=6.0 \mathrm{~Hz}, 2 \mathrm{H}$ ), 1.98 (quintet, $J=6.0 \mathrm{~Hz}, 2 \mathrm{H}$ ), 1.81 (s, $3 \mathrm{H}$ ), 1.13 (s, $9 \mathrm{H}$ ). ${ }^{13} \mathrm{C}\left\{{ }^{1} \mathrm{H}\right\}$ NMR: $\delta$ 215.2, 197.2, 156.9, 14.01, 44.7, 37.3, 31.8, 27.2, 22.5, 22.0. IR (neat, $\mathrm{cm}^{-1}$ ): 2970 , 2925, 1699, 1661, 1423. TLC: $R_{f}=0.13$. Anal. calcd (found) for $\mathrm{C}_{12} \mathrm{H}_{18} \mathrm{O}_{2}: \mathrm{H}, 9.34$ (9.37); C, 74.19 (74.26).

2-Benzoyl-3-methyl-2-cyclohexenone (Table 1, entry 8). ${ }^{1} \mathrm{H}$ NMR: $\delta \quad 7.85-7.83(\mathrm{~m}, 2 \mathrm{H})$, 7.58-7.54 (m, $1 \mathrm{H}), 7.46-7.43(\mathrm{~m}, 2 \mathrm{H}), 2.51(\mathrm{t}, J=6.8 \mathrm{~Hz}, 2 \mathrm{H}), 2.50(\mathrm{t}, J=6.4 \mathrm{~Hz}, 2 \mathrm{H}), 2.13(\mathrm{~m}, 2 \mathrm{H})$, 1.87 (s, $3 \mathrm{H}) .{ }^{13} \mathrm{C}\left\{{ }^{1} \mathrm{H}\right\}$ NMR: $\delta$ 197.5, 192.7, 160.1, 138.1, 137.1, 134.0, 129.4, 129.0, 37.6, 32.3, 22.4, 22.2. TLC: $R_{f}=0.08 .{ }^{1} \mathrm{H}$ NMR data was consistent with the published data. ${ }^{7}$

2-Acetyl-3,4,4-trimethyl-2-cyclohexenone (Table 1, entry 9). Tan solid, mp 51-53 ${ }^{\circ} \mathrm{C} .{ }^{1} \mathrm{H}$ NMR: $\delta 2.48(\mathrm{~d}, J=6.8 \mathrm{~Hz}, 1 \mathrm{H}), 2.46(\mathrm{~d}, J=6.8 \mathrm{~Hz}, 1 \mathrm{H}), 2.29(\mathrm{~s}, 3 \mathrm{H}), 1.86(\mathrm{~d}, J=6.8 \mathrm{~Hz}, 2 \mathrm{H}), 1.83$ (s, $3 \mathrm{H}), 1.19$ (s, $6 \mathrm{H}) .{ }^{13} \mathrm{C}\left\{{ }^{1} \mathrm{H}\right\}$ NMR: $\delta$ 205.8, 196.9, 164.7, 139.5, 37.0, 36.0, 34.7, 32.1, 26.5, 16.8 . IR (neat, $\mathrm{cm}^{-1}$ ): 2964, 2920, 2869, 1709, 1666, 1607, 1547, 1468, 1422, 1376, 1354, 1333, 1308, 1280 , 1211, 1162. TLC: $R_{f}=0.22$. Anal. calcd (found) for $\mathrm{C}_{11} \mathrm{H}_{16} \mathrm{O}_{2}: \mathrm{H}, 8.95$ (8.98); C, 73.30 (73.26).

2-Acetyl-3-methyl-spiro[5.5] undec-2-en-1-one (Table 1, entry 10). ${ }^{1} \mathrm{H}$ NMR: $\delta 2.35$ (t, $J=$ $6.0 \mathrm{~Hz}, 2 \mathrm{H}), 2.28$ (s, $3 \mathrm{H}), 1.89$ (s, $3 \mathrm{H}), 1.87$ (t, $J=6.0 \mathrm{~Hz}, 2 \mathrm{H}), 1.76-1.69$ (m, $2 \mathrm{H}), 1.66-1.59$ (m, $2 \mathrm{H})$, 1.55-1.32 (m, $6 \mathrm{H}) .{ }^{13} \mathrm{C}\left\{{ }^{1} \mathrm{H}\right\}$ NMR: $\delta$ 205.0, 156.7, 43.5, 31.7, 31.6, 30.1, 29.0, 26.1, 21.8, 21.4. IR (neat, $\mathrm{cm}^{-1}$ ): 2925, 2861, 1698, 1659, 1447. TLC: $R_{f}=0.24$. Anal. calcd (found) for $\mathrm{C}_{14} \mathrm{H}_{20} \mathrm{O}_{2}: \mathrm{H}, 9.15$ (9.28); C, 76.33 (76.49). 


\section{References}

1) Doherty, A. M.; Ley, S. V.; Lygo, B.; Williams, D. J. J. Chem. Soc., Perkin Trans. 1 1984, 1371.

2) Hasegawa, T.; Hojo, F.; Yoshioka, M. Bull. Chem. Soc. Jpn. 1990, 63, 2428.

3) Kashimura, S.; Murai, Y.; Ishifune, M.; Masuda, H.; Shimomura, M.; Murase, H.; Shono, T. Acta Chem. Scand. 1999, 53, 949.

4) Smith, A. B.; Levenberg, P. A. Synthesis 1981, 7, 567.

5) Kawashima, M.; Sato, T.; Fujisawa, T. Tetrahedron 1989, 45, 403.

6) Brocard, J.; Moinet, G.; Conia, J. M. Bull. Soc. Chim. Fr. 1973, 5, 1711.

7) Tamura, Y.; Wada, A.; Okuyama, S.; Fukumori, S.; Hayashi, Y.; Gohda, N.; Kita, Y. Chem. Pharm. Bull. 1981, 29, 1312. 